\title{
Multiple Dural Arteriovenous Fistulas Presenting as Pulsatile Tinnitus Treated with External Manual Compression
}

\author{
Se-Joon Oh, Yong-II Chon, Soo-Keun Kong, and Eui-Kyung Goh \\ Department of Otorhinolaryngology and Biomedical Research Institute, Pusan National University Hospital, Busan, Korea
}

$\begin{array}{ll}\text { Received } & \text { May 18, } 2017 \\ \text { Revised } & \text { August 4, } 2017 \\ \text { Accepted } & \text { August 8, } 2017\end{array}$

Address for correspondence

Eui-Kyung Goh, MD, PhD

Department of Otorhinolaryngology

and Biomedical Research Institute,

Pusan National University Hospital,

179 Gudeok-ro, Seo-gu,

Busan 49241, Korea

Tel +82-51-240-7335

Fax +82-51-246-8668

E-mail gohek@pusan.ac.kr
Dural arteriovenous fistula (DAVF) have been treated by transarterial or transvenous embolization, surgery, and radiosurgery. Besides these treatment options, the external compression technique is a non-invasive, low-cost form of treatment. This article reports that a 60-year-old man with DAVF between multiple arterial branches and transverse/sigmoid sinus was treated by repeated external manual compression method.

J Audiol Otol 2017;21(3):156-159

KEY WORDS: Pulsatile tinnitus · Arteriovenous fistula · Therapeutic.

\section{Introduction}

Rare causes of tinnitus include cranial dural arteriovenous fistula (DAVF) which is responsible for $2-20 \%$ of pulsatile tinnitus [1]. DAVF belong to a group of rare vascular malformations, constituting about $10-15 \%$ of intracranial arteriovenous malformations [2]. They are usually small lesions consisting of numerous, minute abnormal connections between dural arterial branches and venous sinuses or veins and are characterized by absence of pathological tangling of vesselthe so-called "nidus" [3].

The current standard treatment of DAVF is endovascular embolization [4]. However, spontaneous occlusions or occlusions following manual compression in DAVF have been reported $[5,6]$. But the methods of manual compression are diverse, either as compression of the carotid artery solitary or as combination of the carotid artery and the jugular vein compression with the theory that induces thrombosing of the fistula [4]. We herein report a case of pulsatile tinnitus from DAVF of multiple branches of posterior and middle meningeal ar-

This is an Open Access article distributed under the terms of the Creative Commons Attribution Non-Commercial License (http://creativecommons.org/licenses/by-nc/4.0/) which permits unrestricted non-commercial use, distribution, and reproduction in any medium, provided the original work is properly cited. tery, basal tentorial artery to transverse-sigmoid venous sinus which was treated by manual compression technique.

\section{Case Report}

A 60-year-old man was referred to our clinic presenting with 14-month history of persisting unilateral pulsatile tinnitus. He had no history of craniotomy, trauma and did not complain various other neurologic symptoms. Otoscopic examination, audiometry, and tympanometry showed normal finding. Physical examination by stethoscope revealed a pulsatile sound behind the left ear, about $2 \mathrm{~cm}$ above the mastoid process. Tinnitus Handicap Inventory (THI) quantitative questionnaire was performed by the patient, which scored 52 points. The patient complained high pitch sound like whistle regularly depend on his position. Manual compression in the left side of the neck and mastoid process led the noise to cease. An initial magnetic resonance imaging (MRI) with magnetic resonance angiography (MRA) demonstrated the suspicious left transverse venous sinus arteriovenous fistula (Fig. 1). Since MRA findings showed a suggestive DAVF, catheter angiography was obtained revealing abnormal vasculture along branches of posterior and middle meningeal artery, basal tentorial artery and early filling of the transverse/sigmoid sinus, 
which confirmed the diagnosis of DAVF (Fig 2). Because there was no risk of neurological complications such as increased intracranial pressure, cerebral hemorrhage, focal neurological symptoms, we decided to wait until the fistula could be occluded spontaneously instead of endovascular embolization. Thus, we tried to compress the neck intermittently to help self occlusion. The patient was educated to conduct the dual compression of the carotid artery and jugular vein with seating position, above the neck carotid bifurcation with 2-4 fingers as a power of the flow sounds disappeared. The daily compression was applied 20-30 times for 10s each time. There were no side effects during manual compression, and the patient did not feel discomfort as he understood the manual compression well. And the patients were followed up every 3 months and the THI score and qualitative questionnaire were surveyed. After 1 month of external manual compression without further treatment, he reported resolution of pulsatile tinnitus and we could not observe any tinnitus sound by stethoscope. THI score decreased to 0 points at 3 months

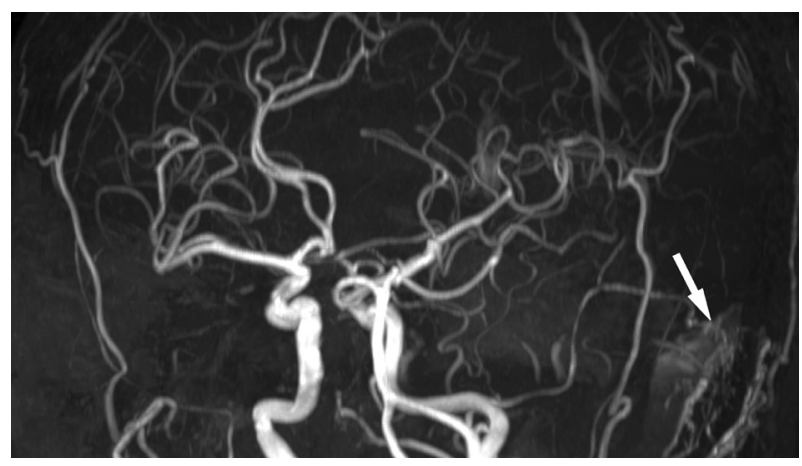

Fig. 1. Left transverse venous sinus AVF fed by multiple branches of posterior meningeal artery of left vertebral artery, basal tentorial artery of left internal carotid artery, and occipital, middle meningeal, and posterior auricular arteries of left external carotid artery (arrow). AVF: arteriovenous fistula. after treatment. And tinnitus did not recurred during 12 month follow up period. The patient was suggested for taking a follow-up imaging after symptom improvement. However, the patient already experienced symptom resolution and did not want further examination, so imaging work-up was not possible.

\section{Discussion}

Patients with pulsatile tinnitus occupy a small (approximately $4 \%$ ) subset of most patients presenting with a tinnitus [7]. Unlike subjective tinnitus, it arises from a mechanical somatosound. And consideration of differential diagnosis for pulsatile tinnitus is important because there is an array of causes that can be identifiable and treatable easily, and many of them are vascular origin problem [8]. DAVF are abnormal arteriovenous shunts between dural arteries and venous sinuses and around the meningeal/cortical venous vessels [8]. Although their etiology is not clearly understood, DAVF have been found in association with venous thrombosis, intracranial surgery, tumor, trauma and congenital anomalies. Depending on their location and the venous drainage pattern, it arises mostly at the transverse, sigmoid, and cavernous sinuses [2].

Imaging modalities for the patients with pulsating tinnitus are necessary to detect the potentially treatable vascular etiologies, and they include computed tomography angiography (CTA), non-enhanced CT of the temporal bone, MRI and MRA, catheter angiogram [9]. CTA is considered as initial imaging examination of choice, because it provides a robust assessment of various intra- and extratemporal vascular pathologies, including DAVF, as well as an excellent description of the bony anatomy of the temporal bone [8]. Temporal bone CT may represent the transosseous vascular shapes, which are highly specific for the diagnosis of DAVF [9]. MRI and MRA have advantages in detecting abnormal vascular pathologies asso-
Fig. 2. 4 Vessel angiogram shows dural arteriovenous fistulas (arrows) fed by multiple branches of arteries.

ciated with the dural AV malformations. CTA and MRI could provide additional findings such as asymmetric arterial supply vascultures, "shaggy" or ill-defined dural sinus margins, or dural sinus occlusion. Among these imaging modalities, catheter angiogram is useful in the configuration of negative noninvasive evaluation with highly clinical suspicious for DAVF and has a role as the golden standard for DAVF diagnosis, characterization and planning treatment method [2]. We explained both MRA and CT angiography to the patient at first. The patient wanted to identify the brain parenchyma with MRI, therefore MRI with MRA was taken and catheter angiography was performed for confirmation. Embolization could be performed, but the fistula was not so large, so we tried to perform compression first and got good results.

The therapeutic methods include conservative monitoring, arterial embolization, transvenous occlusion, surgical excision, and radiation therapy [10]. And in the majority of DAVF, transarterial embolization or by transvenous occlusion with coils or ballons in the presence of multiple feeding vessels offers the best chance for successful treatment [11]. But spontaneous occlusions or occlusion by manual compression have often been reported and the pathophysiology is suggested that the frequent and repeated temporary stasis in the fistula areas will promote the increasing thrombosing of the shunt $[5,6]$. Turbulent arterial blood flow elicted by atherosclerosis probably promotes the thrombosis on the venous side of the DAVF or sinus. Spontaneous occlusions may be the result of the pressure fluctuations similar to those caused by manual compression. Because the wide shunt defects and high flow conditions do not allow thrombus formation, the compression maneuver may be only effective in low flow fistulas [12]. The external manual compression technique for the carotid artery and jugular vein was introduced several years ago as an alternative therapy for DAVF, but the efficacy of this technique was not confirmed due to limited available data [4]. And factors that contribute to its success and the closure and healing of these lesions remain unclear [13]. Moreover, the compression effect, compression time per single session, the compression frequency and total compression duration are not clear. Nevertheless, this technique may lead to fistula occlusion successfully or at least improvement of the pulsating tinnitus. Halbach, et al. [14] stated that $22 \%$ cured completely and the improvement of clinical symptoms were $33 \%$. And Cognard, et al. [15] recommended that the compression technique should be increased in duration from 10 to 30 seconds and the frequency should be increased from four to six times per hour. But patients may experience the neurologic deficits such as acute hemiplegia with loss of consciousness during manual compression, which makes the patient hesitate adapting his or her daily routine to the treatment. Therefore the success rate will somewhat depend on the willingness of the patient to do the frequent compressions continuously. Since the changes in fistula activity and changes in flow sound are closely related to compression techniques, the relationship between the repeated manual compression therapy in our patient and the subsequent, sustained fistula obstruction might be close [4]. External compression technique may stimulate the vagus nerve located in the carotid sheath and thereby induce vasovagal reactions following in cardiac arrhythmias such as sinus bradycardia, atrioventricular block, systole, and syncope. Especially, elderly patients with significant atherosclerotic disease are at increased risk for neurological deficits and stroke from compromised carotid blood flow and dislodged emboli [13]. Although the consensus about the method of the manual compression therapy is not existed, this therapy might give useful opportunity of the improvement due to its noninvasiveness and simplicity.

\section{Acknowledgments}

This work was supported by a 2-year Research Grant of Busan National University.

\section{Conflicts of interest}

The authors have no financial conflicts of interest.

\section{REFERENCES}

1) Waldvogel D, Mattle HP, Sturzenegger M, Schroth G. Pulsatile tinnitus--a review of 84 patients. J Neurol 1998;245:137-42.

2) Gandhi D, Chen J, Pearl M, Huang J, Gemmete JJ, Kathuria S. Intracranial dural arteriovenous fistulas: classification, imaging findings, and treatment. AJNR Am J Neuroradiol 2012;33:1007-13.

3) Gupta A, Periakaruppan A. Intracranial dural arteriovenous fistulas: a review. Indian J Radiol Imaging 2009;19:43-8.

4) Schumacher M, Szczeponik N. Successful treatment of dural AV fistulas by manual compression: a matter of perseverance. Neuroradiology 2007;49:495-8.

5) Chung SJ, Kim JS, Kim JC, Lee SK, Kwon SU, Lee MC, et al. Intracranial dural arteriovenous fistulas: analysis of 60 patients. Cerebrovasc Dis 2002;13:79-88.

6) Kerber CW, Newton TH. The macro and microvasculature of the dura mater. Neuroradiology 1973;6:175-9.

7) Park SN. Objective tinnitus. J Clinical Otolaryngol 2011;22:35-43.

8) Fitzgerald RT, Pollitzer R, Samant RS, Kumar M, Ramakrishnaiah $\mathrm{RH}$, Amole A, et al. Pulsatile tinnitus secondary to a dural arteriovenous fistula. Otol Neurotol 2015;36:e115-6.

9) Narvid J, Do HM, Blevins NH, Fischbein NJ. CT angiography as a screening tool for dural arteriovenous fistula in patients with pulsatile tinnitus: feasibility and test characteristics. AJNR Am J Neuroradiol 2011;32:446-53.

10) Kim S, Byun J, Park M, Lee S. Pulsatile tinnitus with a dural arterio-venous fistula diagnosed by computed tomography-angiography. Korean J Audiol 2013;17:133-7.

11) Berlis A, Klisch J, Spetzger U, Faist M, Schumacher M. Carotid cavernous fistula: embolization via a bilateral superior ophthalmic vein approach. AJNR Am J Neuroradiol 2002;23:1736-8.

12) Endo S, Koshu K, Suzuki J. Spontaneous regression of posterior fossa dural arteriovenous malformation. J Neurosurg 1979;51:715-7.

13) Kai Y, Morioka M, Yano S, Nakamura H, Makino K, Takeshima H, 
et al. External manual carotid compression is effective in patients with cavernous sinus dural arteriovenous fistulae. Interv Neuroradiol 2007;13 Suppl 1:115-22.

14) Halbach VV, Higashida RT, Hieshima GB, Goto K, Norman D, Newton TH. Dural fistulas involving the transverse and sigmoid si- nuses: results of treatment in 28 patients. Radiology 1987;163:443-7. 15) Cognard C1, Gobin YP, Pierot L, Bailly AL, Houdart E, Casasco A, et al. Cerebral dural arteriovenous fistulas: clinical and angiographic correlation with a revised classification of venous drainage. Radiology 1995;194:671-80. 